\title{
Noncommutative Mean Ergodic Theory
}

\author{
Charles RADIN \\ Instituut voor Theoretische Fysica, Universiteit Nijmegen
}

Received February 15, 1971

\begin{abstract}
Sufficient conditions are given to obtain two levels of ergodic behavior for a wide class of dynamical systems. The notion of substate is emphasized and shown to lead to a natural generalization for noncommutative systems of the mean ergodic theorem on $L_{1}$. Applications to the time averaging of certain deviations from thermal equilibrium is mentioned.
\end{abstract}

\section{Introduction}

In previous work $[1,2]$ we obtained regularity properties for functions of time of the form $\langle A\rangle_{t}$ for a wide class of dynamical models. These properties were used to determine the connection between the spectrum of the Hamiltonian of a model and the rate of approach to equilibrium of that model. In the present work we use these results to obtain general ergodic properties for these same models.

Specifically, the models we will be considering are called abstract dynamical systems and consist of a triple $(\mathfrak{H}, \mathfrak{S}, \alpha)$ where: $\mathfrak{A}$ is a $C^{*}$ algebra with unit $I, \mathfrak{S}$ is the set of all states on $\mathfrak{U}$, and $\alpha$ is a time development ${ }^{1}$ of $\mathfrak{A}$, that is, a weakly continuous group representation of $\mathbb{R}$ in the set Aut( $\mathfrak{Q})$ of automorphisms of $\mathfrak{Q}$. (A class of examples used extensively below is the generalized Ising models, defined in Appendix I.)

In order to state the regularity properties referred to above, it is convenient to define the following objects pertaining to an abstract dynamical system $(\mathfrak{A}, \mathfrak{S}, \alpha)$. We denote by $\alpha_{t}$ the image of $t \in \mathbb{R}$ in $\operatorname{Aut}(\mathfrak{U})$ and by $\alpha_{t}^{*}$ its dual map of $\mathfrak{U}^{*}$ onto $\mathfrak{Q}^{*}$. For $A \in \mathfrak{U}, \alpha(A)$ is the function

$$
\alpha(A): t \in \mathbb{R} \rightarrow \alpha_{t}(A) \in \mathfrak{A} .
$$

$\mathfrak{S}_{I}\left(\right.$ resp. $\left.\mathfrak{A}_{I}\right)$ is the set of all $\varrho \in \subseteq$ (resp. $A \in \mathfrak{U}$ ) such that $\alpha_{t}^{*}(\varrho)=\varrho$ (resp. $\alpha_{t}(A)=A$ ) for all $t \in \mathbb{R}$. For $\varrho \in \mathfrak{S}, \mathscr{S}(\varrho)$ is the set $\{\tau \in \mathbb{S} \mid \tau \leqq a \varrho$ for some $a \in \mathbb{R}\}$. If $W(\mathbb{R})$ is the set of all weakly almost periodic functions on $\mathbb{R}$ (defined in Appendix II) then $W(\widetilde{S})$ is the set of all $\varrho \in \mathbb{S}$ such that the function

$$
\varrho[\alpha(A)]: t \in \mathbb{R} \rightarrow \varrho\left[\alpha_{t}(A)\right]
$$

1 The conditions we impose are controversial: see $[19,20]$. The continuity assumption is not essential and is specifically overcome for the main result, Prop. 4. 
belongs to $W(\mathbb{R})$ for all $A \in \mathfrak{A}$. For $\varrho \in \mathfrak{S}, \Pi_{\varrho}$ is the GNS representation of $\mathfrak{A}$ on $\mathscr{H}_{\varrho}$ and $\Phi_{\varrho}$ is the canonical cyclic vector. $n, w$ and $w^{*}$ refer to the norm, weak and weak-* topologies respectively.

The above mentioned regularity properties are now:

Proposition 1. If $(\mathfrak{U}, \mathfrak{S}, \alpha)$ is a generalized Ising model, then $W(\mathfrak{S})=\mathfrak{\subseteq}$.

Proposition 2. If $(\mathfrak{H}, \subseteq, \alpha)$ is an abstract dynamical system, $\varrho \in \mathfrak{S}_{I}$, and $\left\{U_{t} \mid t \in \mathbb{R}\right\}$ is the canonical unitary group representation of $\mathbb{R}$ on $\mathscr{H}_{\bar{Q}}$, then for each $\varrho \in \mathscr{S}(\bar{\varrho})$ there exists a positive linear operator $C_{\varrho} \in \Pi_{\bar{\varrho}}(\mathfrak{U})^{\prime}$ such that

$$
\varrho\left[\alpha_{t}(A)\right]=\left\langle U_{t}^{*} C_{\varrho} \Phi_{\bar{\varrho}}, \Pi_{\bar{\varrho}}(A) \Phi_{\bar{\varrho}}\right\rangle
$$

for all $A \in \mathfrak{A}$ and all $t \in \mathbb{R}$. In particular, $\mathscr{S}(\bar{\varrho}) \subseteq W(\mathfrak{S})$.

It will become apparent below that these two results represent quite different aspects of the ergodic nature of abstract dynamical systems; therefore we will treat them in separate sections.

\section{Weak Ergodicity}

The ergodic property pertinent to Prop. 1 is:

Proposition 3. If $(\mathfrak{A}, \mathfrak{S}, \alpha)$ is an abstract dynamical system and if $\varrho \in W(\mathfrak{S})$ then there exists a unique state $\varrho$ in $\overline{\operatorname{co}\left\{\alpha_{t}^{*}(\varrho) \mid t \in \mathbb{R}\right\}}{ }^{w^{*}} \cap \Im_{I}$. Furthermore,

for all $A \in \mathfrak{A}$.

$$
\tilde{\varrho}(A)=\lim _{T \rightarrow \infty} \frac{1}{T} \int_{0}^{T} \varrho\left[\alpha_{t}(A)\right] d t
$$

The proof is in Appendix III.

We now present three examples which show that certain alternative formulations of Prop. 3 would be improper.

Example 1. Let $\mathscr{H}=L_{2}(\mathbb{R})$ and $\mathfrak{A}=B(\mathscr{H})$. For $A \in \mathfrak{A}$, let $\alpha_{t}(A)$ $=U_{t} A U_{t}^{*}$ where

$$
\left(U_{t} f\right)(\tau)=f(\tau-t) \text { for all } f \in \mathscr{H} .
$$

Let $\theta \in \mathfrak{A}$ be defined by

where

$$
(\theta f)(\tau)=\theta(\tau) f(\tau) \text { for all } f \in \mathscr{H}
$$

$$
\theta(\tau)=\left\{\begin{array}{lll}
1 & \text { if } & \tau \geqq 0 \\
0 & \text { if } & \tau<0
\end{array}\right.
$$

For any $g \in \mathscr{H}$ it is straightforward to check that

$$
\lim _{t \rightarrow \infty}\left\langle g, \alpha_{t}(\theta) g\right\rangle=\langle g, g\rangle
$$


and

$$
\lim _{t \rightarrow-\infty}\left\langle g, \alpha_{t}(\theta) g\right\rangle=0 .
$$

It is then not difficult ${ }^{2}$ to show that if $g \neq 0$ there are many states in $\overline{\operatorname{co}\left\{\alpha_{t}^{*}(\hat{g}) \mid t \in \mathbb{R}\right\}^{w^{*}}} \cap \mathfrak{S}_{I}$, where $\hat{g}$ is the state on $\mathfrak{U}$ defined by

$$
\hat{g}(A)=\langle g, A g\rangle /\|g\|^{2} .
$$

This illustrates the necessity of some restriction such as " $\varrho \in W(\Im)$ " in Prop. 3.

Example 2. Let $(\mathfrak{H}, \Im, \alpha)$ be any generalized Ising model and let $\varrho=\bigotimes_{i} \hat{f}_{i}$ where $\sigma_{x}^{i} f_{i}=f_{i}$ for all $i$. Then

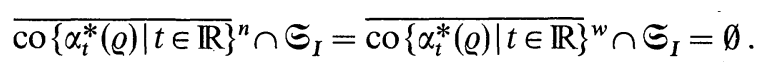

The proof is in Appendix III.

This shows that it is inappropriate, for the general phenomenon apparent from Prop. 1, to use the norm topology on $\mathfrak{U}^{*}$ as is so tempting in ergodic theory. In fact, since it is clearly advantageous to work on a Banach space, it is natural to consider what can be done with $\alpha_{t}: \mathfrak{U} \rightarrow \mathfrak{U}$. The following example eliminates this possibility.

Example 3. Let $(\mathfrak{A}, \mathfrak{S}, \alpha)$ be the generalized Ising model with $v(|j|)$ $=1 /|j|^{2}$. Then

$$
\left.\overline{\operatorname{co}\left\{\alpha_{t}\left(\sigma_{x}^{0}\right) \mid t \in \mathbb{R}\right.}\right\}^{n} \cap \mathfrak{Y}_{I}=\emptyset .
$$

The proof is in Appendix III.

\section{Strong Ergodicity}

If $(\mathfrak{U}, \mathfrak{\subseteq}, \alpha)$ is an abstract dynamical system and $\bar{\varrho} \in \Xi_{I}$, combining Props. 2 and 3 with the fact that $W(\Im)$ is norm closed, it follows that for each $\varrho \in \overline{\mathscr{S}(\bar{\varrho})^{n}}$ there is a unique state $\tilde{\varrho} \in \overline{\left.\operatorname{co}\left\{\alpha_{t}^{*} \varrho\right) \mid t \in \mathbb{R}\right\}} w^{*} \cap \Im_{I}$. However this is not nearly strong enough since:

Proposition 4. If $(\mathfrak{H}, \mathfrak{\Xi}, \alpha)$ is an abstract dynamical system ${ }^{3}$ and $\bar{\varrho} \in \Im_{I}$, then for each $\varrho \in \overline{\mathscr{S}(\bar{\varrho})^{n}}$ there is a unique state $\tilde{\varrho}$ in

Furthermore, $\tilde{\varrho} \in \overline{\mathscr{S}(\bar{\varrho})^{n}}$.

$$
\overline{\operatorname{co}\left\{\alpha_{t}^{*}(\varrho) \mid t \in \mathbb{R}\right\}^{n}} \cap \Im_{I} .
$$

The proof is in Appendix III. Elements of $\overline{\mathscr{S}(\bar{\varrho})^{n}}$ will be called "substates of $\bar{\varrho} "$.

${ }^{2}$ Constructions can be obtained by the method of Prop. 3 using the existence of certain means, as presented for example in $[3 ;$ p. 36].

3 The assumption that $\alpha_{t}$ be weakly continuous in $t$ can be dropped. The only alteration needed in the proof is to redefine the means as states over the $C^{*}$-algebra of all complex bounded functions on $\mathbb{R}$. 
The generality of this result will be manifest from the following two examples.

Example 4 (Classical Dynamics). Let $(\mathfrak{A}, \mathfrak{S}, \alpha)$ be an abstract dynamical system with $\mathfrak{U}$ abelian. Therefore $\mathfrak{U}$ is isomorphic to $C(S)$, the $C^{*}$ algebra of complex continuous functions on the compact Hausdorff space $S$ consisting of the pure states on $\mathfrak{A}$ in the $w^{*}$-topology. If $\varrho \in \Xi_{I} \subseteq \subseteq$, it follows from the Riesz representation theorem [4; IV.6.3] that there is a unique regular measure $\mu_{\bar{\varrho}}$ on $S$ such that

$$
\bar{\varrho}(f)=\int_{S} f(s) d \mu_{\bar{\varrho}} \text { for all } f \in C(S) .
$$

It then follows from the Radon-Nikodym theorem [4; III.10.2] that there is a bijective isometry between the substates of $\varrho$ and the probability densities subordinate to $\mu_{\bar{a}}$ :

$$
\varrho \in \overline{\mathscr{S}(\bar{\varrho})^{n}} \rightarrow f_{\varrho} \in L_{1}^{\mathscr{S}}\left(S, \mu_{\bar{\varrho}}\right) \equiv\left\{f \in L_{1}\left(S, \mu_{\bar{\varrho}}\right) \mid f \geqq 0,\|f\|=1\right\}
$$

such that

$$
\varrho(g)=\int_{S} g(s) f_{\varrho}(s) d \mu_{\bar{\varrho}} \text { for all } g \in C(S) .
$$

From the denseness of $C(S)$ in $L_{1}\left(S, \mu_{\bar{Q}}\right)$ [4; IV.8.19] and the invariance of $\bar{\varrho}$, it is easy to show that $\alpha_{t}^{*}$, as a transformation of $S$ onto $S$, preserves the measure $\mu_{\bar{Q}}$. Thus, as far as abstract dynamical systems are concerned, Prop. 4 is a strict generalization to the noncommutative situation of the mean ergodic theorem on $L_{1}$. In fact it more transparently generalizes the mean ergodic theorem on abstract $L$-spaces as contained for example in [5].

Another case where $\overline{\mathscr{S}(\bar{\varrho})^{n}}$ reduces to a familiar set of states is given in the next example 4 .

Example 5. Let ( $\mathfrak{A}, \mathfrak{S}, \alpha)$ be an abstract dynamical system. Define, for fixed $\phi \in \mathbb{S}$ and $A, B \in \mathfrak{A}$, the functions

$$
\begin{aligned}
& F_{A B}: t \in \mathbb{R} \rightarrow \phi\left[B \alpha_{t}(A)\right] \in \mathbb{C}, \\
& G_{A B}: t \in \mathbb{R} \rightarrow \phi\left[\alpha_{t}(A) B\right] \in \mathbb{C} .
\end{aligned}
$$

Clearly $F_{A B}$ and $G_{A B}$ are bounded continuous functions of $\mathbb{R}$, and can therefore be considered as elements of $\mathscr{S}^{\prime}(\mathbb{R})$, the tempered distributions on $\mathbb{R}$. Defining the function

$$
e_{\beta}: t \in \mathbb{R} \rightarrow e^{\beta t} \in \mathbb{C}
$$

we say that $\phi$ is a KMS state for inverse temperature $\beta \neq 0$ if

$$
\hat{F}_{A B}=e_{\beta} \hat{G}_{A B}
$$

${ }^{4}$ The theory of KMS states does not require the strong assumptions in the time development which we imposed: see e.g. [19]. 
where ${ }^{\wedge}$ is the Fourier transformation on $\mathscr{S}^{\prime}(\mathbb{R})$. By setting $B=I$ in (1) and using standard techniques ${ }^{5}$ it follows that a KMS state is a priori time invariant ${ }^{6}$. If now it is further assumed ${ }^{7}$ that the KMS state $\phi$ satisfies the condition

$$
\left\{x \in \mathfrak{A} \mid \phi\left(x^{*} x\right)=0\right\}=\{0\}
$$

then ${ }^{8}$ it can be shown that $\Phi_{\phi}$ is a cyclic vector for $\Pi_{\phi}(\mathfrak{A})^{\prime}[22,7]$. Therefore $^{9}{\overline{\mathscr{S}}(\phi)^{n}}^{n}=v(\phi)$, the set of all vector states from $\mathscr{H}_{\phi}$. And since $\overline{\mathscr{S}(\phi)^{n}}$ is convex [4; V.2.4 and V.1.2] we have $\overline{\mathscr{S}(\phi)}^{n}=v(\phi)=\overline{\operatorname{co}\{v(\phi)}^{n}$, the set of all density matrix states on $\Pi_{\phi}(\mathfrak{H})$.

At this point we would like to mention how this example complements a certain result of Emch. It was pointed out in [8] that the time average of a density matrix state need not be a density matrix state, i.e. that if an initial state is ultraweakly continuous with respect to some representation $\Pi$ of $\mathfrak{U}$, then its time average need not be ultraweakly continuous with respect to $\Pi$. However, if $\varrho$ is a KMS state with ${ }^{8}$ trivial left ideal then it follows from example 5 that the time average of a density matrix state on $\Pi_{\varrho}(\mathfrak{Q})$ is uniquely defined and is a density matrix state on $\Pi_{Q}(\mathfrak{l})$.

\section{Conclusion and Summary}

We have shown above that the two classes of states, the weakly almost periodic and the substates of any equilibrium state, are well behaved in the sense that they exhibit ergodic behavior in the weak-* and norm topologies respectively. The notion of substates is seen to be a natural and useful noncommutative generalization of the probability density states of Gibbs; in the case of thermal equilibrium ${ }^{4}$ it coincides with the concept of density matrix states. While, therefore, our results apply to those states of an abstract dynamical system often called "local perturbations" from thermal equilibrium, we will give some explicit results in this direction in a future publication.

Acknowledgements. This work was carried out under an NSF Postdoctoral Fellowship at the 1970 Summer Workshop for Statistical Mechanics at Montana State University, which support and hospitality is gratefully acknowledged. It is also a pleasure to express my indebtedness for helpful discussions with G. G. Emch, G. Gallavotti and H. J. F. Knops.

\footnotetext{
${ }^{5}$ For example combine Prop. 4.6.4 with Example 4.11.1 and Thrm. 4.11.1 of [6].

${ }^{6}$ See e.g. [21].

7 This assumption can be replaced by the stronger one that $\mathfrak{A}$ is simple $[22,7]$.

${ }^{8}$ It was pointed out to the author that this assumption is unnecessary.

${ }^{9}$ We note that such cyclicity is also necessary for this identification; see the corollary of theorem $G$ in [16].
} 


\section{Appendix I}

To each site $i$ in the $v$-dimensional lattice $\mathbb{Z}^{v}$ we associate a twodimensional complex Euclidean space $\mathbb{C}_{i}^{2}$. Let $F$ be the set of all finite subsets of $\mathbb{Z}^{v}$ partially ordered by inclusion. Then for each $\Lambda \in F$, consider the direct product space $\otimes \mathbb{C}_{i}^{2}$ of Murray and von Neumann [9], and let $\mathfrak{A}(\Lambda)$ be defined as $B\left(\bigotimes_{i \in \Lambda} \underset{i}{i} \mathbb{C}_{i}^{2}\right)$. Then $\mathfrak{A}$ is defined as the inductive limit of the $C^{*}$-algebras $\mathfrak{A}(\Lambda)$ in the sense of Takeda [10]. To simplify notation we identify $\mathfrak{A}(\Lambda)$ with its image in $\mathfrak{A}$ and also with $G L\left(2^{N(\Lambda)}, \mathbb{C}\right)$, where $N(\Lambda)$ is the number of sites in $\Lambda$. For example $\sigma_{x}^{i} \in \mathfrak{A}(i) \cong \mathfrak{A}$ for the Pauli matrix $\sigma_{x}$.

For $\Lambda \in F, \Lambda \neq \emptyset$, define

$$
H_{\Lambda}=\frac{1}{2} \sum_{(j, k) \in \Lambda \times \Lambda} v(|j-k|) \sigma_{z}^{j} \sigma_{z}^{k}
$$

where $v$ is a real function of the Euclidean distance $|j|$ such that $v(0)=0$ and $\sum_{j \in \mathbb{Z}^{\nu}}|v(|j|)|<\infty$. It can be shown ${ }^{10}$ that the net $\left\{\alpha_{t}^{\Lambda}(A) \mid \Lambda \in F\right\}$, where

$$
\alpha_{t}^{\Lambda}(A)=\exp \left[i H_{\Lambda} t\right] A \exp \left[-i H_{\Lambda} t\right]
$$

has a norm limit, $\alpha_{t}(A)$, in $\mathfrak{A}$ for all $A \in \mathfrak{A}$ and $t \in \mathbb{R}$. The set $\left\{\alpha_{t} \mid t \in \mathbb{R}\right\}$ forms a strongly continuous group of automorphisms of $\mathfrak{U}$, so that $(\mathfrak{U}, \mathfrak{S}, \alpha)$ is an abstract dynamical system, called a generalized Ising model.

If $a \in \mathbb{Z}^{v}$, the natural mapping

$$
\tau_{a}: A \in \mathfrak{A}(\Lambda) \rightarrow \tau_{a}(A) \in \mathfrak{A}\left(\Lambda_{a}\right),
$$

where $\Lambda_{a}=\{b+a \mid b \in \Lambda\}$, extends to an automorphism of $\mathfrak{A}$ denoted by the same symbol $\tau_{a} . \varrho \in \subseteq$ is said to be translation invariant if $\varrho\left[\tau_{a}(A)\right]$ $=\varrho(A)$ for all $A \in \mathfrak{A}$ and all $a \in \mathbb{Z}^{v}$.

Given a normalized vector $f_{i}$ in each $\mathbb{C}_{i}^{2}$ we denote by $\hat{f}_{i}$ the vector state on $\mathfrak{A}(i)$ associated to $f_{i}$, and by $\underset{i}{\bigotimes} f_{i}$ the product state on $\mathfrak{A}$.

We should add that the above class of models is a very special case of the Robinson spin models [23]. A recent generalization without some of the special characteristics of Robinson's models is contained in [20].

\section{Appendix II}

Let $C B(\mathbb{R})$ be the $C^{*}$-algebra of complex bounded continuous functions on $\mathbb{R}$. We call the states on $C B(\mathbb{R})$ "means". The means $\mu_{t}$ defined for $t \in \mathbb{R}$ by

$$
\mu_{t}: f \in C B(\mathbb{R}) \rightarrow f(t) \in \mathbb{C}
$$

${ }^{10}$ For this and other details concerning the time development of these models see [1]. See also [23]. 
are needed below. For $\tau \in \mathbb{R}$, let $R_{\tau}$ be the operator on $C B(\mathbb{R})$ defined by

$$
\left(R_{\tau} f\right)(t)=f(t+\tau) \text { for all } f \in C B(\mathbb{R}) .
$$

A mean $\eta$ is said to be invariant if

$$
\eta\left[R_{\tau} f\right]=\eta(f) \text { for all } f \in C B(\mathbb{R}) \text { and } \tau \in \mathbb{R} .
$$

The set $W(\mathbb{R})$ of weakly almost periodic functions on $\mathbb{R}$ is defined as the subset of $C B(\mathbb{R})$ consisting of those $f$ such that $\left\{R_{\tau} f \mid \tau \in \mathbb{R}\right\}$ is conditionally weakly compact. $W(\mathbb{R})$ is a norm closed subspace of $C B(\mathbb{R})$ [11], and from this it is easy to show that $W(\mathbb{S})$ is a norm closed subset of $\mathfrak{S}$. It is an important theorem of Eberlein's [11] that all invariant means $\eta$ coincide on $W(\mathbb{R})$ and may be computed as

$$
\eta(f)=\lim _{T \rightarrow \infty} \frac{1}{T} \int_{0}^{T} f(t) d t .
$$

If $\eta$ is a mean and $(\mathfrak{A}, \mathfrak{S}, \alpha)$ is an abstract dynamical system then for $\varrho \in \subseteq$ the functional $\eta \varrho$ on $\mathfrak{A}$ defined by

$$
\eta \varrho(A)=\eta\{\varrho[\alpha(A)]\} \quad \text { for all } \quad A \in \mathfrak{A}
$$

is a state on $\mathfrak{A}[12]$. If $\eta$ is an invariant mean then $\eta \varrho \in \mathfrak{S}_{I}$.

\section{Appendix III}

Proof of Prop. 3. The proposition follows immediately from the following two lemmas and the theorem of Eberlein referred to in Appendix II.

Lemma 1. $\eta \varrho \in \overline{\operatorname{co}\left\{\alpha_{t}^{*}(\varrho) \mid t \in \mathbb{R}\right\}} w^{*}$ for all $\varrho \in \subseteq$ and all means $\eta$.

Proof. From [3; pp. 1-2] we know that any mean $\eta$ is the $w^{*}$-limit of some net of "finite means" $m_{\alpha}$, i.e. where the $m_{\alpha}$ are elements of the convex hull of the states $\mu_{t}, t \in \mathbb{R}$. Therefore, for each $A \in \mathfrak{A}$

$$
\eta \varrho(A) \equiv \eta\{\varrho[\alpha(A)]\}=\lim _{\alpha} m_{\alpha}\{\varrho[\alpha(A)]\}=\lim _{\alpha} m_{\alpha} \varrho(A) .
$$

Since it is clear that $m_{\alpha} \varrho \in \operatorname{co}\left\{\alpha_{t}^{*}(\varrho) \mid t \in \mathbb{R}\right\}$, the proof is completed.

Lemma 2. If $\varrho \in \subseteq$ and $\varrho \in \overline{\operatorname{co}\left\{\alpha_{t}^{*}(\varrho) \mid t \in \mathbb{R}\right\}} w^{*}$, then there exists a mean $\eta$ such that $\bar{\varrho}=\eta \varrho$. Furthermore, if $\bar{\varrho} \in \Xi_{I}$ then $\eta$ can be chosen invariant.

Proof. We only prove the case $\bar{\varrho} \in \Xi_{I}$, the other case following from obvious simplifications in the proof. From the form of $\bar{\varrho}$, there exists a 
net of states $\left\{\varrho_{\gamma} \mid \gamma \in G\right\}$ such that $\varrho_{\gamma} \in \operatorname{co}\left\{\alpha_{t}^{*}(\varrho) \mid t \in \mathbb{R}\right\}$ and $\varrho_{\gamma} \frac{w^{*}}{\gamma} \bar{\varrho}$. Since

$$
\left[\alpha_{t}^{*}\left(\varrho_{\gamma}\right)\right](A) \equiv \varrho_{\gamma}\left[\alpha_{t}(A)\right] \underset{\gamma}{\longrightarrow} \bar{\varrho}\left[\alpha_{t}(A)\right] \equiv\left[\alpha_{t}^{*}(\bar{\varrho})\right](A)=\bar{\varrho}(A)
$$

for all $A \in \mathfrak{A}$ and $t \in \mathbb{R}$, we have that $\alpha_{t}^{*}\left(\varrho_{\gamma}\right) \frac{w^{*}}{\gamma} \bar{\varrho}$ for all $t \in \mathbb{R}$. Now from the form of $\varrho_{\gamma}$, for each $\gamma \in G$ there exist $P_{\gamma}$ numbers $t_{n}(\gamma) \in \mathbb{R}$ and $P_{\gamma}$ numbers $\beta_{n}(\gamma) \in[0,1]$ such that

$$
\varrho_{\gamma}=\sum_{n=1}^{P_{\gamma}} \beta_{n}(\gamma) \alpha_{t_{n}(\gamma)}^{*}(\varrho) .
$$

Consider the net of finite means $\left(M_{\gamma}, G\right)$ where

$$
M_{\gamma} \equiv \sum_{n=1}^{P_{\gamma}} \beta_{n}(\gamma) \mu_{t_{n}(\gamma)} .
$$

Note that $M_{\gamma} \varrho=\varrho_{\gamma}$. Since the set of means is $w^{*}$-compact, there exists a mean $\eta$ and a subnet ${ }^{11}\left(N_{\delta}, D\right)$ of $\left(M_{\gamma}, G\right)$ such that $N_{\delta} \frac{w^{*}}{\delta} \eta$. Furthermore, defining the mean $R_{\tau} \eta^{\prime}$ by:

$$
\left(R_{\tau} \eta^{\prime}\right)(f)=\eta^{\prime}\left(R_{\tau} f\right) \text { for all } f \in C B(\mathbb{R}),
$$

for all means $\eta^{\prime}$, we have that

$$
R_{\tau} N_{\delta} \underset{w^{*}}{\stackrel{w^{*}}{\longrightarrow}} R_{\tau} \eta \quad \text { for all } \tau \in \mathbb{R}
$$

Now we know that

$$
M_{\gamma}\{\varrho[\alpha(A)]\} \underset{\gamma}{\longrightarrow} \bar{\varrho}(A) \text { for all } A \in \mathfrak{U} .
$$

This implies by the definition of subnet that

$$
N_{\delta}\{\varrho[\alpha(A)]\} \underset{\delta}{\longrightarrow} \bar{\varrho}(A) \text { for all } A \in \mathfrak{U} .
$$

Therefore also

$$
R_{\tau} N_{\delta}\{\varrho[\alpha(A)]\} \underset{\delta}{\longrightarrow} \bar{\varrho}(A) \text { for all } A \in \mathfrak{U} \quad \text { and } \quad \tau \in \mathbb{R} .
$$

Therefore $\left(R_{\tau} \eta\right)(\varrho)=\varrho$ for all $\tau \in \mathbb{R}$. Defining $\eta_{m}(f)=m[R \eta(f)]$ where $m$ is any fixed invariant mean, all we need to show is that $\eta_{m}$ is an invariant mean since clearly $\eta_{m} \varrho=\bar{\varrho}$. But this is very easy and we omit the details. q.e.d.

Constructions for Example 2. We need the following lemma.

Lemma 3. $\left\{\alpha_{t}^{*}(\varrho) \mid t \in \mathbb{R}\right\}$ are all pure and translation invariant. Furthermore, for $t \neq t^{\prime}, \alpha_{t}^{*}(\varrho) \neq \alpha_{t^{\prime}}^{*}(\varrho)$ implies that $\alpha_{t}^{*}(\varrho)$ is unitarily inequivalent to $\alpha_{t^{\prime}}^{*}(\varrho)$.

Proof. The pureness and translation invariance are obvious. Now assume that $\alpha_{t}^{*}(\varrho)$ is unitarily equivalent to $\alpha_{t^{\prime}}^{*}(\varrho)$ for fixed $t \neq t^{\prime}$. Then

11 For definitions and properties of nets and subnets, see e.g. [13]. 
there exists a unitary $u \in \mathfrak{A}$ such that $\left[\alpha_{t}^{*}(\varrho)\right](D)=\left[\alpha_{t^{\prime}}(\varrho)\right]\left(u^{*} D u\right)$ for all $D \in \mathfrak{A}[14]$. Now given $\varepsilon>0$ there exists a volume $\Lambda \in F$ and $A \in \mathfrak{A}(\Lambda)$ such that

$$
\|u-A\|<\varepsilon \quad \text { and } \quad\|A\|<2 .
$$

Then since for all $B \in \mathfrak{A}$ we have

$$
\left\|u^{*} B u-A^{*} B A\right\|=\left\|u^{*} B(u-A)+\left(u^{*}-A^{*}\right) B A\right\| \leqq 3 \varepsilon\|B\|,
$$

we have

$$
\left\|\left[\alpha_{t}(\varrho)\right](\cdot)-\left[\alpha_{t^{\prime}}(\varrho)\right]\left(A^{*} \cdot A\right)\right\| \leqq 3 \varepsilon .
$$

Now given a volume $\Lambda^{\prime} \in F$, by a sufficient translation by $a \in \mathbb{Z}^{v}$ we can get $\Lambda_{a}^{\prime}$ disjoint from $\Lambda$. Using the fact that $\left\|A^{*} A-I\right\|<3 \varepsilon$ we have then

$$
\left|\left[\alpha_{t}^{*}(\varrho)\right]\left[\tau_{a}(B)\right]-\left[\alpha_{t^{\prime}}^{*}(\varrho)\right]\left[\tau_{a}(B)\right]\right| \leqq 6 \varepsilon\|B\|
$$

for all $B \in \mathfrak{U}\left(\Lambda^{\prime}\right)$. Therefore using the first part of the lemma,

$$
\left|\left[\alpha_{t}(\varrho)\right](B)-\left[\alpha_{t^{\prime}}(\varrho)\right](B)\right| \leqq 6 \varepsilon\|B\|
$$

for all $B \in \mathfrak{A}\left(\Lambda^{\prime}\right)$. Therefore $\alpha_{t}^{*}(\varrho)=\alpha_{t^{\prime}}^{*}(\varrho)$. q.e.d.

Now assume that there exists $\varrho \in \overline{c o}\left\{\alpha_{t}^{*}(\varrho) \mid t \in \mathbb{R}^{n} \cap \Im_{I}\right.$. Then there exists a sequence $\varrho_{i} \in \operatorname{co}\left\{\alpha_{t}^{*}(\varrho) \mid t \in \mathbb{R}\right\}$ such that $\varrho_{i} \underset{i}{i} \tilde{\varrho}$. From the lemma we can find a sequence (perhaps finite) of $t_{n} \in \mathbb{R}$ such that $\left\{\alpha_{t_{n}}^{*}(\varrho) \mid n\right.$ $=1,2, \ldots\}$ are pairwise unitarily inequivalent and $\varrho_{i} \in \operatorname{co}\left\{\alpha_{t_{n}}^{*}(\varrho) \mid n\right.$ $=1,2, \ldots\}$ for all $i$. Let $\left\{a_{n}\right\}$ be a sequence (of the same cardinality) of strictly positive numbers such that $\Sigma a_{n}=1$, and define

$$
\varrho_{0}=\Sigma a_{n} \alpha_{t_{n}}^{*}(\varrho) \text {. }
$$

Consider $\oplus \Pi_{\alpha_{t_{n}}(\varrho)}$ on $\oplus \mathscr{H}_{\alpha_{t_{n}}^{*}(\varrho)}$, and $\Phi=\left\{\Phi_{n} \mid n=1,2, \ldots\right\} \quad$ with $\Phi_{n}=\sqrt{a_{n}} \Phi_{\alpha t_{n}(\varrho)}$. By the transitivity theorem [14] $\Phi$ is a cyclic vector for $\oplus \Pi_{\alpha t_{n}(\varrho)}$. From [15; p. 245], $\oplus \Pi_{\alpha_{t_{n}}(\varrho)}$ is unitarily equivalent to $\Pi_{\varrho_{0}}$. Now since $\varrho_{i} \in \operatorname{co}\left\{\alpha_{t_{n}}^{*}(\varrho) \mid n=1,2, \ldots\right\}, \varrho_{i}$ is a vector state from $\oplus H_{\alpha_{n}(\varrho)}$ for all $i$. Therefore [16] $\varrho$ is also a vector state from $\oplus H_{\alpha t_{n}(\varrho)}$, and let $\Psi$ be a corresponding vector. Clearly

$$
\Psi=\Sigma b_{n} \Phi_{\alpha *_{n}(\varrho)}
$$

for some sequence $b_{n} \in B\left(\mathscr{H}_{\alpha_{n}^{*}(\varrho)}\right)$. Then $\tilde{\varrho}$ is a sum of inequivalent pure states. Therefore $\tilde{\varrho}$ gives a type I representation since the commutant of $\Pi_{\bar{\varrho}}(\mathfrak{Q})$ is $\oplus\left\{\lambda I_{n}\right\}$ which is abelian. But $\eta \varrho$ has been computed [1] to be $\bigotimes_{i} T R_{i}$, and it is known [17] that this state gives a type II representation.

This contradiction shows that $\overline{\operatorname{co}\left\{\alpha_{t}^{*}(\varrho) \mid t \in \mathbb{R}^{n}\right.} \cap \cap \mathcal{S}_{I}=\emptyset$. From [4; V.3.13], $\overline{\operatorname{co}\left\{\alpha_{t}^{*}(\varrho) \mid t \in \mathbb{R}\right\}^{n}}=\overline{\operatorname{co}\left\{\alpha_{t}^{*}(\varrho) \mid t \in \mathbb{R}\right\}^{w}}$, so the demonstration is complete. 
Constructions for Example 3. In [1] there is a construction of a state $\varrho$ and a net $\left\{\varrho_{\beta} \mid \beta \in B\right\}$ such that $\varrho_{\beta} \stackrel{w^{*}}{\longrightarrow} \varrho$ and

$$
\begin{array}{rlrl}
\lim _{T \rightarrow \infty} \frac{1}{T} \int_{0}^{T} \varrho_{\beta}\left[\alpha_{t}\left(\sigma_{x}^{0}\right)\right] d t=0 & \text { for all } & \beta \in B, \\
\varrho\left[\alpha_{t}\left(\sigma_{x}^{0}\right)\right] & =1 & \text { for all } & t \in \mathbb{R} .
\end{array}
$$

From [11] we can replace (4) by

$$
E\left[\lim _{\alpha} M_{\alpha}\left\{\varrho_{\beta}\left[\alpha\left(\sigma_{x}^{0}\right)\right]\right\}\right]=0 \text { for all } \beta \in B
$$

where $E[f]=f(0)$ for all $f \in C B(\mathbb{R})$ and where $\left\{M_{\alpha} \mid \alpha \in A\right\}$ is the net of convex combinations of the operators $\left\{R_{t} \mid t \in \mathbb{R}\right\}$ on $C B(\mathbb{R})$ with the ordering: $M_{\alpha} \leqq M_{\beta}$ if there exists $\gamma \in A$ such that $M_{\beta}=M_{\alpha} M_{\gamma}$. Now if $D \in \overline{\operatorname{co}\left\{\alpha_{t}\left(\sigma_{x}^{0}\right) \mid t \in \mathbb{R}\right\}^{n}} \cap \mathcal{S}_{I}$ it follows from [11] that

$$
D=\operatorname{norm} \lim _{\alpha} M_{\alpha}^{\prime}\left(\sigma_{x}^{0}\right)
$$

where $\left\{M_{\alpha}^{\prime}\right\}$ are the corresponding convex combinations of the $\left\{\alpha_{t} \mid t \in \mathbb{R}\right\}$. Note that

Therefore

$$
\varrho_{\beta}\left[M_{\alpha}^{\prime}\left(\sigma_{x}^{0}\right)\right]=E\left\{M_{\alpha}\left[\varrho_{\beta}\left\{\alpha\left(\sigma_{x}^{0}\right)\right\}\right]\right\} .
$$

$$
\begin{aligned}
1 & =\lim _{\alpha} \varrho\left[M^{\prime}\left(\sigma_{x}^{0}\right)\right]=\lim _{\alpha} \lim _{\beta} \varrho_{\beta} M_{\alpha}^{\prime}\left(\sigma_{x}^{0}\right) \\
& =\lim _{\beta} \lim _{\alpha} \varrho_{\beta} M_{\alpha}^{\prime}\left(\sigma_{x}^{0}\right)=\lim _{\beta} \lim _{\alpha} E\left[M_{\alpha}\left\{\varrho_{\beta}\left[\alpha\left(\sigma_{x}^{0}\right)\right]\right\}\right] \\
& =0
\end{aligned}
$$

where the third equality follows from (6). This contradiction demonstrates the assertion.

Proof of Prop.4. For $\varrho \in \mathscr{S}(\bar{\varrho})$ we know from Prop. 2 that there exists $C_{\varrho} \in \Pi_{\bar{\varrho}}(\mathfrak{l})^{\prime}$ such that

$$
\left[\alpha_{t}^{*}(\varrho)\right](A)=\left\langle U_{t}^{*} C_{\varrho} \Phi_{\bar{\varrho}}, \Pi_{\bar{\varrho}}(A) \Phi_{\bar{\varrho}}\right\rangle
$$

for all $A \in \mathfrak{A}$ and $t \in \mathbb{R}$. From [18] we know that there exists a vector $\Psi$ in $\mathscr{H}_{\bar{\varrho}}$ such that

$$
\Psi \in \overline{\operatorname{co}\left\{U_{t}^{*} C_{\varrho} \Phi_{\bar{\varrho}} \mid t \in \mathbb{R}\right\}^{n}} \cap\left\{\omega \in H_{\bar{\varrho}} \mid U_{t}^{*} \omega=\omega \text { for all } t \in \mathbb{R}\right\} .
$$

Therefore there exists a sequence $\Psi_{m} \in \operatorname{co}\left\{U_{t}^{*} C_{\varrho} \Phi_{\bar{\varrho}} \mid t \in \mathbb{R}\right\}$ such that

$$
\left\|\Psi-\Psi_{m}\right\|_{m} 0 \text {. }
$$

It is clear from their form that the functionals on $\mathfrak{A}$ :

$$
\varrho_{m}(\cdot)=\left\langle\Psi_{m}, \Pi_{\bar{\varrho}}(\cdot) \Phi_{\bar{\varrho}}\right\rangle
$$


are states and in fact belong to $\operatorname{co}\left\{\alpha_{t}^{*}(\varrho) \mid t \in \mathbb{R}\right\}$. From (8) it is easy to show that $\varrho_{m} \frac{n}{m} \varrho_{0}$ where

$$
\varrho_{0}(\cdot) \equiv\left\langle\Psi, \Pi_{\bar{\varrho}}(\cdot) \Phi_{\bar{\varrho}}\right\rangle .
$$

Since $\subseteq$ is norm closed, $\varrho_{0} \in \subseteq$. From (7) we see that

$$
\begin{aligned}
{\left[\alpha_{t}^{*}\left(\varrho_{0}\right)\right](\cdot) } & =\left\langle\Psi, U_{t} \Pi_{\bar{\varrho}}(\cdot) U_{t}^{*} \Phi_{\bar{\varrho}}\right\rangle \\
& =\left\langle\Psi, \Pi_{\bar{\varrho}}(\cdot) \Phi_{\bar{\varrho}}\right\rangle=\varrho_{0}
\end{aligned}
$$

for all $t \in \mathbb{R}$. This completes the case for $\varrho \in \mathscr{S}(\bar{\varrho})$. It follows from [11] however that the set of all $\varrho \in \mathbb{S}$ such that there exists

$$
\left.\varrho \in \overline{\operatorname{co}\left\{\alpha_{t}^{*}(\varrho) \mid t \in \mathbb{R}\right.}\right\}^{n} \cap \Xi_{I}
$$

is norm closed so that the first part of the proof is complete. To show that $\tilde{\varrho} \in{\overline{\mathscr{S}}(\bar{\varrho})^{n}}^{n}$ we again consider two cases. The case where $\rho \in \mathscr{S}(\bar{\varrho})$ is obvious since if $\varrho \leqq a \bar{\varrho}$ then $\alpha_{t}^{*} \varrho \leqq a \bar{\varrho}$ so that $\phi \leqq a \varrho$ for any $\phi \in \overline{\operatorname{co}\left\{\alpha_{t}^{*}(\varrho) \mid t \in \mathbb{R}\right\}^{w^{*}}}$ and so $\tilde{\varrho} \leqq a \bar{\varrho}$. For the case $\varrho \in \overline{\mathscr{S}(\bar{\varrho})^{n}}$ we use lemma 2 to see that $\tilde{\varrho}=\eta \varrho$ for some invariant mean $\eta$. Then the result follows from the following lemma.(Uniqueness follows trivially from $\S 11$ of [18].)

Lemma 4. The mapping $\eta: \varrho \in \subseteq \rightarrow \eta \varrho \in \subseteq$ is continuous when $\subseteq$ is in the norm topology, for any mean $\eta$.

Proof. Let $\varrho_{m}, \varrho \in \Xi$, with $\varrho_{m} \frac{n}{m} \varrho$. Then

$$
\varrho_{m}\left[\alpha_{t}(A)\right] \underset{m}{\longrightarrow} \varrho\left[\alpha_{t}(A)\right]
$$

uniformly in $t \in \mathbb{R}$ and in $A \in \mathfrak{A r}_{1}$, the unit ball of $\mathfrak{A}$. Therefore

$$
\eta\left\{\varrho_{m}[\alpha,(A)]\right\} \underset{m}{\rightarrow} \eta\{\varrho[\alpha,(A)]\}
$$

uniformly in $A \in \mathfrak{A}_{1}$. Therefore $\eta \varrho_{m} \frac{n}{m} \rightarrow \varrho$. q.e.d.

\section{References}

1. Radin, C.: J. Math. Phys. 11, 2945 (1970).

2. - Thesis, Univ. of Rochester, 1970.

3. Greenleaf,F.P.: Invariant means on topological groups. New York: van NostrandReinhold Co. 1969.

4. Dunford, N., Schwartz,J.T.: Linear operators, Part I. New York: Interscience 1957.

5. Namioka, I.: Studia Math. 29, 63 (1967).

6. Horvath, J.: Topological vector spaces and distributions. Reading, Massachusetts: Addison-Wesley Publishing Company 1966.

7. Kastler, D., Pool, J.C.T., Poulsen, E. T.: Commun. math. Phys. 12, 175 (1969).

8. Emch, G. G.: J. Math. Phys. 7, 1413 (1966).

9. Murray, F.J., von Neumann, J.: Ann. Math. 37, 116 (1936).

10. Takeda,Z.: Tôhoku Math. J., Ser. 2 (7), 67 (1955).

11. Eberlein, W.: Trans. Am. Math. Soc. 67, 217 (1949). 
12. Emch, G. G., Knops, H.J.F., Verboven, E.J.: Commun. math. Phys. 7, 164 (1968).

13. Kelley,J.L.: General topology. New York: D. van Nostrand 1955.

14. Glimm, J., Kadison, R. V.: Pacific. J. Math. 10, 547 (1960).

15. Naimark, M. A.: Normed rings, trans. L.F.Boron. Groningen: P. Noordhoff N.V. 1964.

16. Kadison, R.V.: Trans. Am. Math. Soc. 103, 304 (1962).

17. Størmer, E.: J. Functional Anal. 3, 48 (1969).

18. Alaoglu, L., Birkhoff, G.: Ann. Math. 41, 293 (1940).

19. Dubin, D., Sewell, G.: J. Math. Phys. 11, 2990 (1970).

20. Ruskai, M.: Commun. math. Phys. 20, 193 (1971).

21. Winnink, M.: Groningen, Thesis (1968).

22. Haag, R., Hugenholtz, N., Winnink, M.: Commun. math. Phys. 5, 215 (1967).

23. Robinson, D.: Commun. math. Phys. 7, 337 (1968).

Charles Radin

Institute of Theoretical Physics

University of Nijmegen

Nijmegen, The Netherlands 